\title{
Object Detection by Point Feature Matching using Matlab
}

\author{
${ }^{1}$ Faishal Badsha, ${ }^{2}$ Rafiqul Islam, ${ }^{3,{ }^{*}}$ Mohammad Farhad Bulbul \\ ${ }^{1}$ Department of Mathematics and Statistics, Bangladesh University of Business and Technology, \\ (BUBT), Dhaka, Bangladesh \\ ${ }^{2}$ Mathematics Discipline, Khulna University (KU), Khulna, Bangladesh \\ ${ }^{3}$ Department of Mathematics, Jessore University of Science and Technology (JUST), \\ Jessore, Bangladesh \\ faishalbadsha0703@gmail.com; mrislam66@yahoo.com; farhad@just.edu.bd
}

\begin{abstract}
Objects detection is an important part in image processing field. For detection object at first we take a photo called test image and then detect some region or point on the image and compare it with reference image. For this task, we use an algorithm that is used in many computer vision applications and is also considered very fast by compared to others. This algorithm can detect and describe local features for any interest object and extract features or descriptor points from it and compare it with the features that extracted from original image. Matching process is done among features and decision is made based on similar features found. This algorithm is called Speeded up Robust Features (SURF) algorithm. In this paper, we use the SURF algorithm which can detect the position of the interest object in original image by using geometric transform. This object capturing method works best for objects that exhibit in a cluttered texture patterns. When a part of object is occluded by other objects in the scene, in this case we use this algorithm to find reference image.
\end{abstract}

Keywords: Object capture, Point Feature Matching, Detection, SURF" algorithm, Cluttered Scenes, matching technique, geometric primitives.

\section{Introduction}

In image processing, point feature detection is an effective method to detect a specified target in a cluttered scene. Regarding specified, this method is to detect one specific object instead of that kind of objects. For instance, by using this method, we are able to recognize one specific object in a cluttered scene. The algorithm of this method is, simply speaking, based on comparing and analyzing point correspondences between the reference target image and the cluttered scene image. Object detection systems are increasingly used in the fields of industrial automation and home robotics. Real-time object learning and detection are important and challenging tasks. Among the application fields that drive development in this area, robotics especially has a strong need for computationally efficient approaches, as autonomous systems continuously have to adapt to a changing and unknown environment, and to learn and recognize new objects. For such time-critical applications, point feature matching is an attractive solution because new objects can be easily learned online, in contrast to statistical-learning techniques that require many training samples. Our approach is related to recent and efficient matching methods and more particularly to, which consider only images and their gradients to detect objects. The method of object detection works best for objects that exhibit nonrepeating texture patterns, which give rise to unique feature matches. This technique is not likely to work well for uniformly colored objects, or for objects containing repeating patterns. For detecting 
objects of a particular category we use here Speeded Up Robust Features "SURF" algorithm. There is another way to detect object called "SIFT" (Scale Invariant Feature Transform) algorithm .But in our study we use here Speeded Up Robust Features "SURF" algorithm because "SURF" is more better than " SIFT ". Speeded Up Robust Features "SURF" algorithm is a local feature and descriptor algorithm that can be used in many application such as object recognition , "SURF" use much larger number of features descriptor from origin image which can reduce contribution of the errors caused by local variation in the average of all feature matching. SURF can robustly recognize and identify objects in origin images even in case of clutter and partial occlusion because "SURF" has feature descriptor which is invariant to scale, partial variant in illumination changes and orientation. The process of Speeded UP Robust Features "SURF" algorithm can be divided into three main steps. First step is "Detection step", in this step interest points are selected at distinctive locations in the origin image, such as corners, blobs etc and this process must be robustly. Second step is "Description step", in this step interest points should have unique identifiers does not depend on features scale and rotations which are called descriptor, the information of interest points represented by descriptor which are vectors that contain information about the points itself and the surroundings. Third step is "Matching step", in this step descriptor vectors are compared between the object image and the new input or origin image, the matching score is calculated based on the distance between vectors e.g. Euclidian distance and the sign of Laplacian. This algorithm can be used in many application such as recognize and locate of objects, track objects, face recognition, make 3D scenes. In this paper we used enhanced SURF algorithm for detect and locate the objects if it's found in origin image.

\section{Comparison of SIFT and SURF}

\subsection{SIFT algorithm overview}

SIFT (Scale Invariant Feature Transform) algorithm proposed by Lowe in 2004 to solve the image rotation, scaling, and affine deformation, viewpoint change, noise, illumination changes, also has strong robustness. The SIFT algorithm has four main steps: (1) Scale Space Extrema Detection, (2) Key point Localization, (3) Orientation Assignment and (4) Description Generation. The first stage is to identify location and scales of key points using scale space extrema in the DoG (Difference-of Gaussian) functions with different values of $\sigma$, the DoG function is convolved of image in scale space separated by a constant factor $k$ as in the following equation.

$$
D(x, y, \sigma)=(G(x, y, k \sigma)-G(x, y, \sigma) \times I(x, y))
$$

Where, $\mathrm{G}$ is the Gaussian function and $\mathrm{I}$ is the image. Now the Gaussian images are subtracted to produce a DoG, after that the Gaussian image subsample by factor 2 and produce DoG for sampled image. A pixel compared of $3 \times 3$ neighborhood to detect the local maxima and minima of $D(x, y, \sigma)$. In the key point localization step, key point candidates are localized and refined by eliminating the key points where they rejected the low contrast points. In the orientation assignment step, the orientation of key point is obtained based on local image gradient. In description generation stage is to compute the local image descriptor for each key point based on image gradient magnitude and orientation at each image sample point in a region centered at key point; these samples building 3D histogram of gradient location and orientation; with $4 \times 4$ array location grid and 8 orientation bins in each sample. That is 128-element dimension of key point descriptor. 


\subsection{SURF algorithm overview}

SURF (Speed Up Robust Features) algorithm, is base on multi-scale space theory and the feature detector is base on Hessian matrix. Since Hessian matrix has good performance and accuracy. In image $I, X=(x, y)$ is the given point, the Hessian matrix $H(x, \sigma)$ in $X$ at scale $\sigma$, it can be define as

$$
H(x, \sigma)=\left[\begin{array}{ll}
L_{x x}(x, \sigma) & L_{x y}(x, \sigma) \\
L_{y x}(x, \sigma) & L_{y y}(x, \sigma)
\end{array}\right]
$$

Where $L_{x x}(x, \sigma)$ is the convolution result of the second order derivative of Gaussian filter $\frac{\partial^{2}}{\partial x^{2}} g(\sigma)$ with the image $I$ in point $X$ and similarly for $L_{x y}(x, \sigma)$ and $L_{y y}(x, \sigma)$ SURF creates a "stack" without 2:1 down sampling for higher levels in the pyramid resulting in images of the same resolution. Due to the use of integral images, SURF filters the stack using a box filter approximation of second-order Gaussian partial derivatives. Since integral images allow the computation of rectangular box filters in near constant time. In Figure 1 Show the Gaussian second orders partial derivatives in $y$-direction and $x y$-direction.In descriptors, SIFT is good performance compare to other descriptors. The proposed SURF descriptor is based on similar properties. The first step consists of fixing a reproducible orientation based on information from a circular region around the interest point. And second construct a square region aligned to the selected orientation, and extract the SURF descriptor from it. In order to be invariant to rotation, it calculate the Haar-wavelet responses in $\mathrm{x}$ and $\mathrm{y}$ direction shown in figure 2 .
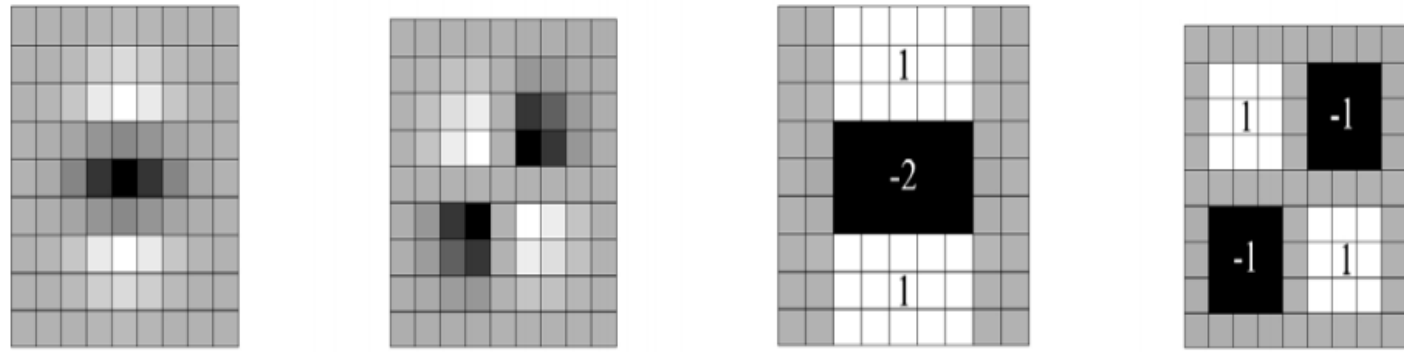

Figure 1.The Gaussian second orders partial derivatives in $y$-direction and $x y$-direction.

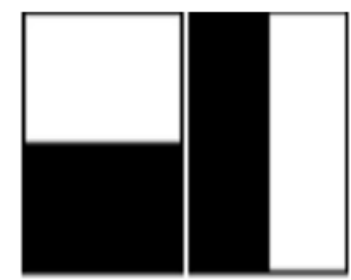

Figure 2. Haar wavelet types used for SURF

\subsection{SIFT and SURF}

SIFT and SURF algorithms employ slightly different ways of detecting features. SIFT builds an image pyramids, filtering each layer with Gaussians of increasing sigma values and taking the difference. On the other hand, SURF creates a "stack" without 2:1 down sampling for higher levels in the pyramid resulting in images of the same resolution [6]. Due to the use of integral images, SURF filters the stack using a box filter approximation of second-order Gaussian partial derivatives, since integral images allow the computation of rectangular box filters in near constant time. In key point matching step, the nearest neighbor is defined as the key point with minimum Euclidean distance for the invariant descriptor vector. 


\section{Point feature technique}

\subsection{Point feature matching technique}

Definition of feature: Feature is defined as an "interesting" part of an image and features are used as a starting point for many computer vision algorithms. The desirable property for a feature detector is repeatability: whether or not the same feature will be detected in two or more different images of the same scene. Feature detection is computationally expensive and there are time constraints, a higher level algorithm may be used to guide the feature detection stage, so that only certain parts of the image are searched for features.

\subsection{Types of image features}

(i) Edges

(ii) Corners / interest points

(iii) Blobs / regions of interest or interest points

(iv) Ridges

\subsection{Description of algorithm}

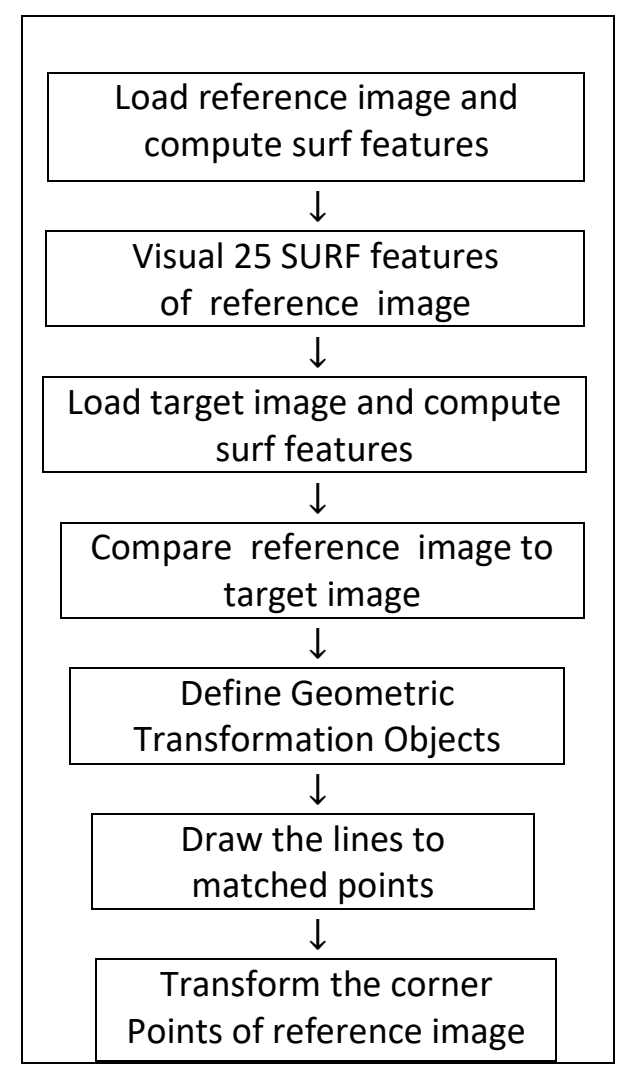

\section{Experiments and Analysis}

\subsection{Experiment}

Let us consider an image of a house as a target image which has different part in its structure including a car a gate and a tree (figure: 4). So it is said that the target image is a cluttered scene. We try to detect the tree part from the reference image. In this side tree part is the target image. 


\subsubsection{Reference image}

Let us first take the reference image shown in figure-1.

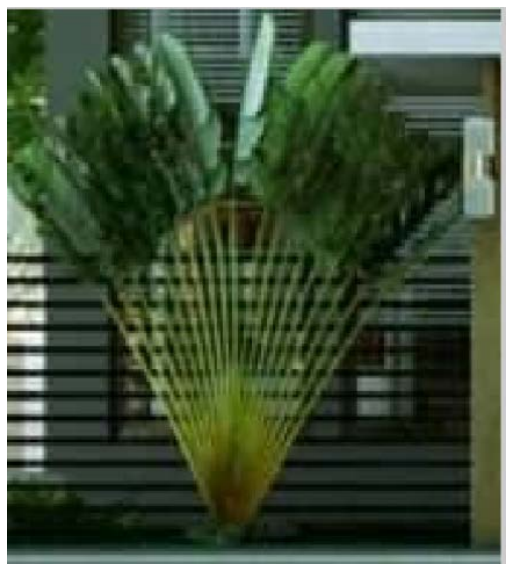

Figure-1: Reference image (tree of target image).

\subsubsection{Feature point detect}

Now detect about 100 feature point on the reference image

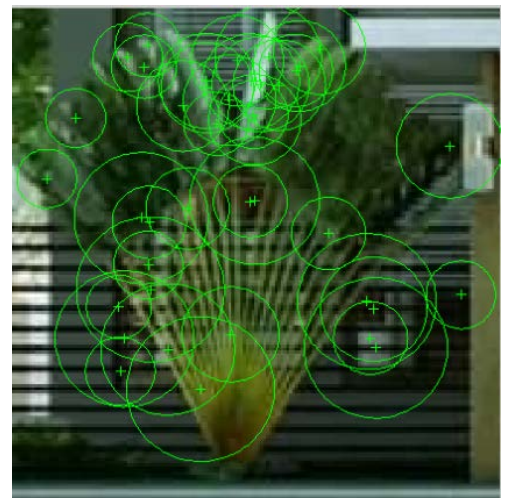

Figure-2: 100 feature point on the reference image (tree of target image).

\subsubsection{Strong feature point detect}

Take the visual 25 SURF feature of the reference image which is shows 25 strong feature point on the reference image.

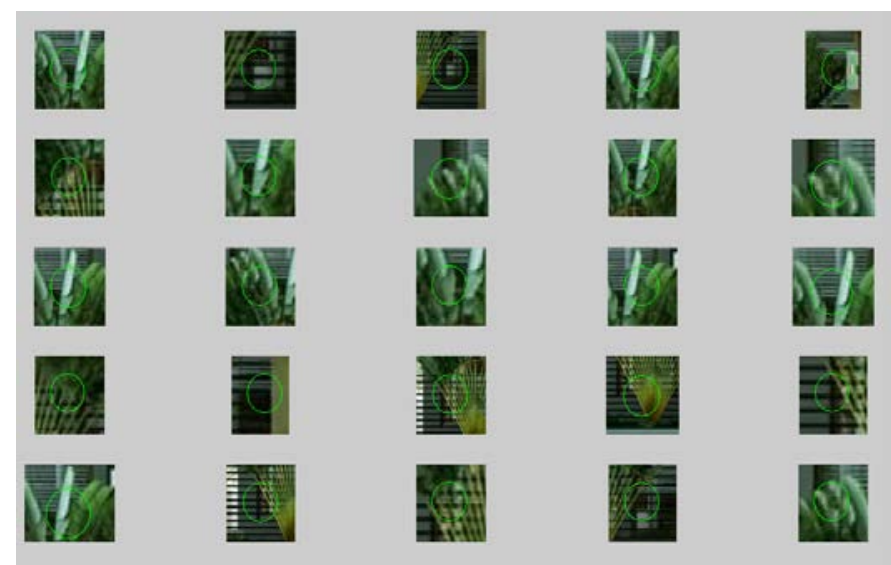

Figure-3: Visual 25 SURF feature of the reference image. 


\subsubsection{Target image}

Now take the image of a house as a target image which is considered as cluttered scene.

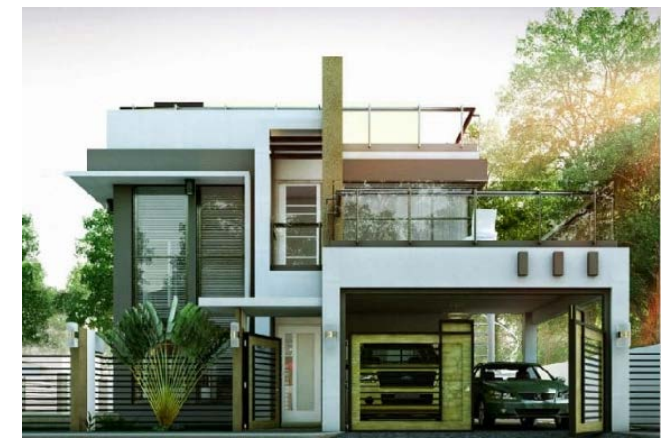

Figure-4: Image of cluttered scene

\subsubsection{Feature point of target image}

After taking the target image, detect about 300 feature point on the target image.

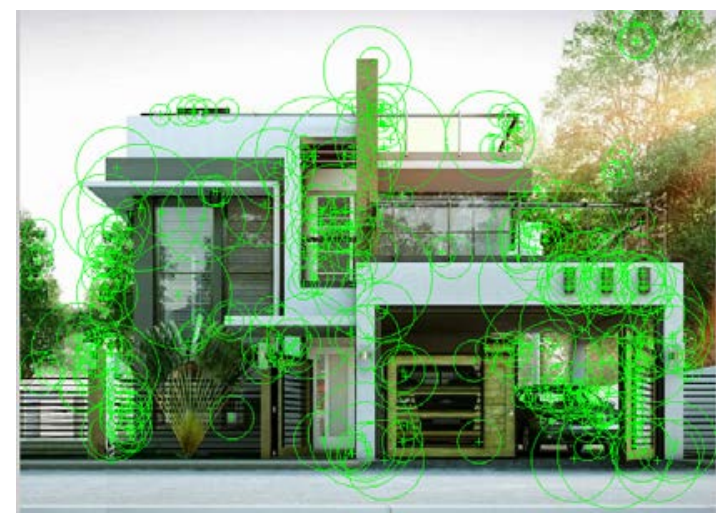

Figure-5: Detect about 300 feature point on the target image.

\subsubsection{Compare reference and target image}

After detecting the feature point of reference image and target image we compare the reference image to target image by comparing its point feature.

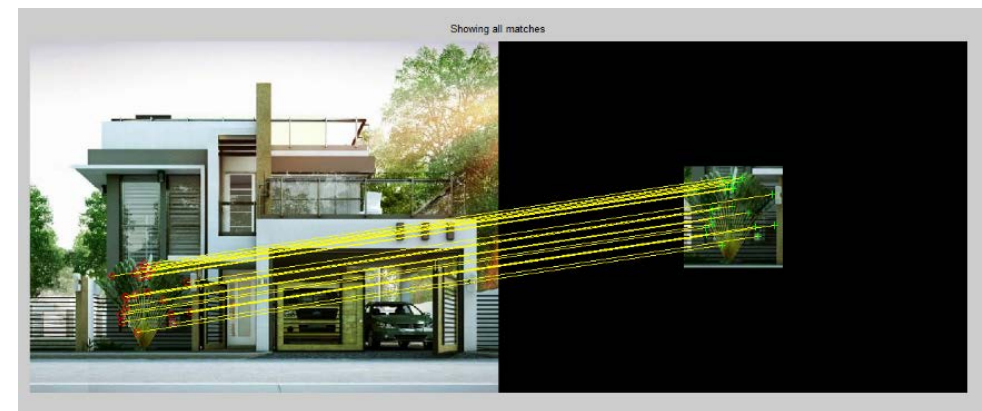

Figure-6: Showing all matching between target image and reference image. 
Faishal Badsha, Rafiqul Islam, Mohammad Farhad Bulbul; Object Detection by Point Feature Matching using Matlab, Advances in I mage and Video Processing, Volume 6 No 6, December (2018); pp: 22-29

\subsubsection{Draw line from target to reference}

After matching all the feature point we draw line to the matched point

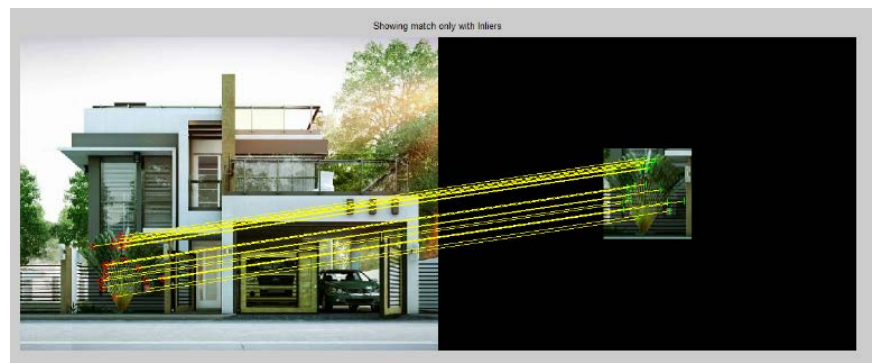

Figure-7: Showing match only with line.

\subsubsection{Corner point transform}

Now transform the corner point to the target image, this will show where the reference image is located in the target image.

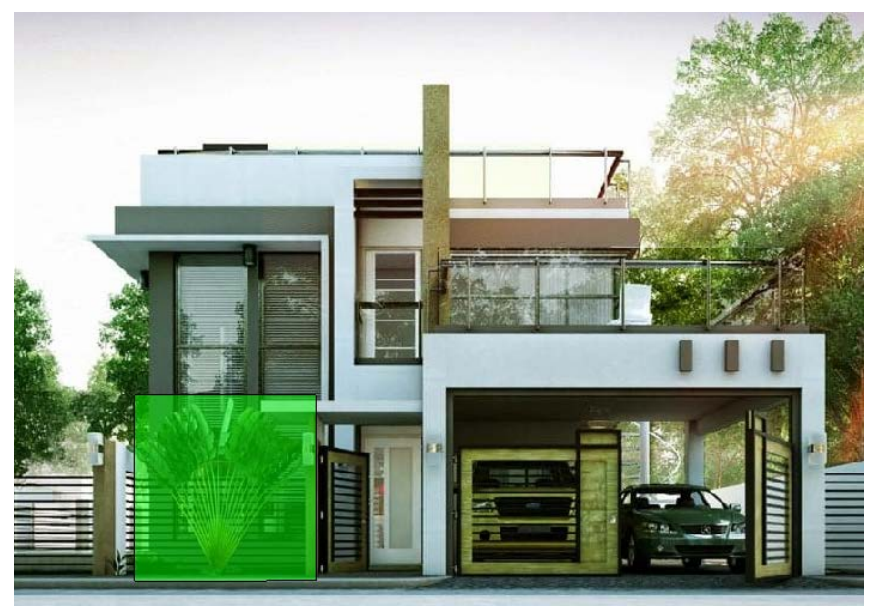

Figure-7: Corner point to the target image

\section{Results and discussions}

At first we load the reference image and target image. After loading, we compute the full SURF feature descriptors from the reference image and target image and match them. Before matching reference image to target image we select 25 strong feature of reference image. After matching all the feature point we draw line to the matched point. Now transform the corner point to the target image, this will show where the reference image is located in the target image. Features are matched in images and object is detected and recognized.

\section{Conclusion}

Object detection is an important tools of image processing. Two previous technique of object detection are segmentation of blob analysis and template matching. But the two process does not work well for complicated image. In our previous study we detect an object in two dimensional image. In this paper, we described a detection method for a three-dimensional image with a complicated background. The method detects an object by comparing its feature point. This feature point may be its edge, corner, blobs etc. Regulation is not the very important issue for SURF feature detection. The proposed algorithm shows that object detection could be improved using SURF features. Our approach can be used in many IT sector as artificial intelligence, robotic sector for a search task in real environment. 


\section{REFERENCES}

[1] Object Detection in a Cluttered Scene Using Point Feature Matching.Object detection in a cluttered scene using point feature matching - MATLAB \& Simulink, Math Works.

[2] Lowe,D.(2004). Distinctive Image Features from Scale-Invariant Key points.International journal of computer vision, pp. 1-28.

[3] Vidyadharan, V., and Surendran, S. (2012).Automatic Image Registration using SIFT-NCC. Specialissue of international journal of computer applications, pp.29-32.

[4] Juan, L., andGwun, O.A Comparison of SIFT, PCA-SIFT and SURF.International journal of image processing (IJIP), 3(4), 143-152.

[5] Bay, H.,Tuytelaars, T., and Gool,L., V.SURF: Speeded Up Robust Features. pp. 1-14

[6] zhan-long,Y., andbao-long, G. (2008). Image Mosaic Based On SIFT. International conference on intelligent information hiding and multimedia signal processing, pp:1422-1425.

[7] Bay,H,. Tuytelaars, T., \&Van,G., L.(2006). SURF: Speeded Up Robust Features. $9^{\text {th }}$ European conference on computer vision. 